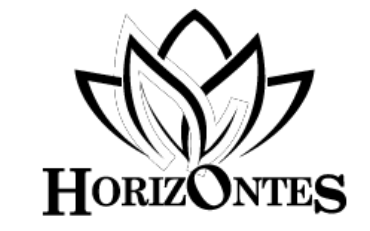

www.revistahorizontes.org
Horizontes. Revista de Investigación en Ciencias de la Educación Https://doi.org/10.33996/revistahorizontes.v4i16.124 Octubre - Diciembre 2020 Volumen 4 / No. 16 ISSN: $2616-7964$ ISSN-L: $2616-7964$ pp. $381-397$

\title{
Estrategia metodológica para elevar el rendimiento académico de los estudiantes en la asignatura investigación educativa
}

\author{
Methodological strategy to raise the academic performance of students in the \\ subject educational research
}

\section{Estratégia metodológica para elevar o desempenho acadêmico dos alunos na disciplina pesquisa educacional \\ ARTÍCULO DE INVESTIGACIÓN}

\author{
Rafael Chamaca Cruz \\ rchamaca2019@gmail.com \\ ORCID: 0000-0002-5784-0997
}

ALSIE Consultores Pedagógicos S.R.L., Bolivia

Recibido 17 de noviembre 2020 | Arbitrado y aceptado 11 de diciembre 2020 | Publicado en diciembre 2020

\section{RESUMEN}

Este artículo presenta resultados del estudio sobre el rendimiento académico de los estudiantes en la asignatura de Investigación Educativa, con el objetivo de contribuir estrategias metodológicas para elevar el rendimiento académico. Los sujetos de la investigación fueron conformados por 284 estudiantes universitarios matriculados en la gestión 2019, carrera Ciencias de la Educación de la Universidad Pública de El Alto, cuya muestra seleccionada corresponde a 142 estudiantes. El abordaje metodológico se ha desarrollado desde una perspectiva dialéctica y los métodos empíricos de encuesta, observación y análisis de contenido. Los resultados revelaron que el $86,3 \%$ de los estudiantes tienen un bajo rendimiento académico en la asignatura de Investigación Educativa; esta situación tiene que ver con los siguientes factores: dificultades de aprendizaje, enfoque de enseñanza tradicional y la influencia de las condiciones socioculturales.

Key words: Proceso enseñanza aprendizaje; rendimiento académico; investigación educativa y estrategia metodológica

\section{ABSTRACT}

This article presents results of the study on the academic performance of students in the subject of Educational Research, with the aim of contributing methodological strategies to improve academic performance. The research subjects were made up of 284 university students enrolled in the 2019 management, Education Sciences career of the Public University of El Alto, whose selected sample corresponds to 142 students. The methodological approach has been developed from a dialectical perspective and the empirical methods of survey, observation and content analysis. The results revealed that $86.3 \%$ of the students have a low academic performance in the Educational Research subject; this situation has to do with the following factors: learning difficulties, traditional teaching approach and the influence of sociocultural conditions.

Palabras clave: Teaching-learning process; academic performance; educational research and methodological strategy 


\section{RESUMO}

Este artigo apresenta resultados do estudo sobre o desempenho acadêmico de alunos na disciplina de Pesquisa Educacional, com o objetivo de contribuir com estratégias metodológicas para a melhoria do desempenho acadêmico. Os sujeitos da pesquisa foram constituídos por 284 estudantes universitários matriculados na carreira de gestão 2019 em Ciências da Educação da Universidade Pública de El Alto, cuja amostra selecionada corresponde a 142 alunos. A abordagem metodológica foi desenvolvida a partir de uma perspectiva dialética e dos métodos empíricos de levantamento, observação e análise de conteúdo. Os resultados revelaram que $86,3 \%$ dos alunos apresentam baixo desempenho acadêmico na disciplina de Pesquisa Educacional; esta situação tem a ver com os seguintes fatores: dificuldades de aprendizagem, abordagem tradicional de ensino e influência das condições socioculturais.

Palavras-Chave: Processo de ensinoaprendizagem; rendimento acadêmico; pesquisa educacional e estratégia metodológica

\section{INTRODUCCIÓN}

La educación superior universitaria constituye uno de los derechos fundamentales para toda persona y la sociedad íntegra. La sociedad en general y los sectores populares, particularmente, consideran como una estrategia para el desarrollo y superación de la pobreza, puesto que a través de la formación profesional el ser humano encuentra diferentes oportunidades para ser instrumento del desarrollo.

Pero adversamente, en todo proceso de formación profesional, se presentan diferentes problemáticas como la baja calidad educativa, condiciones inadecuadas, persistencia de los paradigmas tradicionales, aplicación de metodologías inapropiadas, el fracaso académico, discriminación sociocultural y otros problemas. En el caso concreto de este estudio se ha identificado el problema del bajo rendimiento académico de los estudiantes en la asignatura de Investigación Educativa correspondiente al desarrollo académico de la carrera Ciencias de la Educación de la Universidad Pública de El Alto en Bolivia. La relación causal de este problema no se limita a la capacidad o motivación del estudiante, sino que conlleva otros factores, como ser: la situación la socioeconómica, procedencia cultura de los estudiantes, el enfoque didáctico tradicional, la carencia de nuevas estrategias metodológicas, la percepción de la asignatura como difícil y otros.

Esta investigación está delimitada en el contexto sociocultural aimara porque la Universidad Pública de la ciudad de El Alto está ubicada en un área de asentamiento poblacional de migrantes de las provincias con una trascendencia histórica colonial con mucha dependencia y condiciones adversas; pero en los últimos años, la población alteña se ha constituido en actores protagónicos de las transformaciones sociales y políticas. En este contexto, la carrera Ciencias de la Educación, tiene el propósito principal de formar profesionales con el desarrollo de una consciencia crítica y capacidad de proponer soluciones científicas para transformar la sociedad.

Por consiguiente, el propósito de la investigación es desarrollar competencias investigativas haciendo uso estratégico de las dinámicas interactivas; por lo que, se delimita como objetivo proponer una estrategia metodológica para elevar el rendimiento académico de los estudiantes en la asignatura de Investigación Educativa de la Carrera Ciencias de la Educación de la Universidad Pública de El Alto. 
MÉTODO

El abordaje metodológico de la investigación, en su dimensión teórica, fue desarrollada desde una perspectiva dialéctica porque "'no solo analiza en el contenido del objeto o campo de acción aquellos componentes constituyentes del mismo, sino que determina las contradicciones presentes en éste; es decir establece la relación que determina la igualdad y la diferencia y la oposición entre los polos o componentes con un grado u otro de antagonismo, pero a la vez, con una posibilidad y necesidad de síntesis." (Álvarez de Zayas, 2016, p. 374).
Por otra parte, se utilizó los métodos empíricos de encuesta, observación y análisis de contenido porque constituyen "procedimientos prácticos con el objeto y los medios de investigación que permitieron revelar las características fundamentales y relaciones esenciales del objeto que son accesibles a la contemplación sensorial." (Álvarez de Zayas y Sierra, 2019, p. 80).

Las técnicas e instrumentos (Tabla 1) utilizados según tipo de estudio fueron las siguientes:

Tabla 1. Técnicas e instrumentos del estudio

\begin{tabular}{|c|c|c|}
\hline TIPO & TÉCNICAS & INSTRUMENTOS \\
\hline $\begin{array}{l}\text { Estudio } \\
\text { Iantitativo }\end{array}$ & $\begin{array}{l}\text { - Encuesta sobre rendimiento académico } \\
\text { - Revisión documental de las } \\
\text { calificaciones }\end{array}$ & $\begin{array}{l}\text { - Cuestionario sobre el rendimiento } \\
\text { académico } \\
\text { - Guía de revisión documental de las } \\
\text { calificaciones }\end{array}$ \\
\hline $\begin{array}{l}\text { Estudio } \\
\text { ualitativo }\end{array}$ & $\begin{array}{l}\text { - Observación en el aula } \\
\text { - Análisis de contenido de redacciones }\end{array}$ & $\begin{array}{l}\text { - Guía de observación en el aula } \\
\text { - Instrucción de redacción bajo el tema } \\
\text { "Mi rendimiento académico". }\end{array}$ \\
\hline
\end{tabular}

A partir de la delimitación del objeto de estudio, el proceso de investigación se centró en la operacionalización de variables, determinación de instrumentos, aplicación a nivel piloto, adecuación de los instrumentos, trabajo de campo, análisis e interpretación de datos y elaboración de la propuesta.

- En la operacionalización de variables se han precisado tres dimensiones: cognitiva, afectiva y procedimental.

- Se han determinado cuatro instrumentos: Cuestionario, guía de revisión documental, guía de observación en aula y redacción bajo el tema "Mi rendimiento académico".
- Se ha realizado la aplicación piloto con el propósito de validar instrumentos y su posterior adecuación

- En el trabajo de campo se han aplicado los instrumentos precisados para la obtención de datos sobre el rendimiento académico en la asignatura Investigación Educativa.

- El análisis e interpretación de datos han permitido corroborar resultados y visualizar las alternativas de solución al problema investigado.

- A partir de la constatación del problema, se ha elaborado la propuesta de estrategia metodológica para contribuir en la mejora del rendimiento académico en el campo de la asignatura Investigación Educativa. 
Los instrumentos se han validado según los criterios de viabilidad (preguntas entendibles y aceptables), fiabilidad (consistencia lógica y libre de errores) y constructo (coherencia teórica). El cuestionario y guía de observación han sido validados a través la estrategia de pilotaje; por otra parte, los instrumentos de guía de revisión documental y la instrucción de redacción, han sido validados simplemente tomando en cuenta la experiencia del sistema de calificaciones y la adaptación de los criterios teóricos existentes.

La descripción de las principales características de los instrumentos utilizados se señala a continuación:

- La encuesta sobre rendimiento académico se ha precisado en 5 ítems que implica los siguientes criterios fundamentales: nivel de dificultad en el aprendizaje de las asignaturas en vigencia, nivel de dificultad en el aprendizaje de la asignatura Investigación Educativa, aspectos favorables y desfavorables que inciden en el aprendizaje, actividades académicas que realizan los estudiantes, factores que influyen en el aprendizaje de la asignatura Investigación Educativa.

- En la revisión documental de las calificaciones o el análisis del registro de notas se han tomado los parámetros de aprobación en la escala de 51 a 100 puntos, los cuales están clasificados en tres rangos: 51-66 bajo, 67-83 medio y 83.5-100 alto.

- La guía de observación en el aula, corresponde al registro de información durante el desarrollo de las clases académicas ejecutado por el docente respectivo, cuyos parámetros implican la presencia de las condiciones didácticas en los siguientes criterios: dispositivos materiales, interacción docente, presentación de la clase, postura, expresión oral, uso de recursos audiovisuales, habilidades persuasivas, técnicas interactivas, tipo o estilo de docencia, tipo de estudiantes, retroalimentación y tareas prácticas.

- El instrumento de redacción consiste en la instrucción de modo que los estudiantes elaboren una redacción libre sobre el tema "mi rendimiento académico" con el propósito de analizar los contenidos de tal redacción, cuyos indicadores han sido adoptados del instrumento utilizado en la obra de Carbonell (2009) titulada: "Relación entre la motivación profesional del docente y la satisfacción de los estudiantes", en el que refiere los principales indicadores de análisis de contenido agrupados en los siguientes niveles: Inferior, medio y superior. El nivel inferior se caracteriza por una motivación externa o extrínseca, insatisfacción con la profesión y una actuación caracterizada de pasividad o dependencia; el nivel medio se caracteriza por una motivación interna o intrínseca, pero refleja una satisfacción contradictoria o ambivalente y una actuación conductual relativamente activa; y en el nivel superior se refleja una motivación predominantemente interna o intrínseca, plena de satisfacción y una posición activa relativo a las tareas académicas.

Estos instrumentos fueron aplicados en el siguiente orden: primero la revisión documental de las calificaciones, luego la aplicación del cuestionario sobre el rendimiento académico, seguido por la elaboración de redacciones por los estudiantes $y$ complementado por las observaciones en el aula. 
La población objeto de esta investigación ha sido conformado por 284 estudiantes universitarios matriculados en los semestres tercero y cuarto la gestión académica 2019, que asisten habitualmente a las clases académicas y que en su mayoría son procedentes de las familias y sectores poblacionales aimaras.

La muestra seleccionada fue del $50 \%$ de la población, es decir los instrumentos se han aplicado a 142 estudiantes registrados en la asignatura Investigación Educativa de la carrera Ciencias de la Educación.

\section{RESULTADOS}

Los resultados del estudio se describen según la aplicación de las técnicas referidas: encuesta sobre el rendimiento académico, revisión documental de las calificaciones, observación en aula durante las clases y el análisis de contenido a través de las redacciones que elaboraron los propios estudiantes.

\section{Resultados cuantitativos según encuesta}

Entre los resultados cuantitativos más relevantes se han recopilado datos que tienen que ver con los siguientes indicadores: actividades académicas, nivel de dificultad de aprendizaje y factores que inciden en el rendimiento académico de los estudiantes en la asignatura de Investigación Educativa.

En la figura 1 se puede observar los datos referidos a las actividades académicas que más realizan los estudiantes.

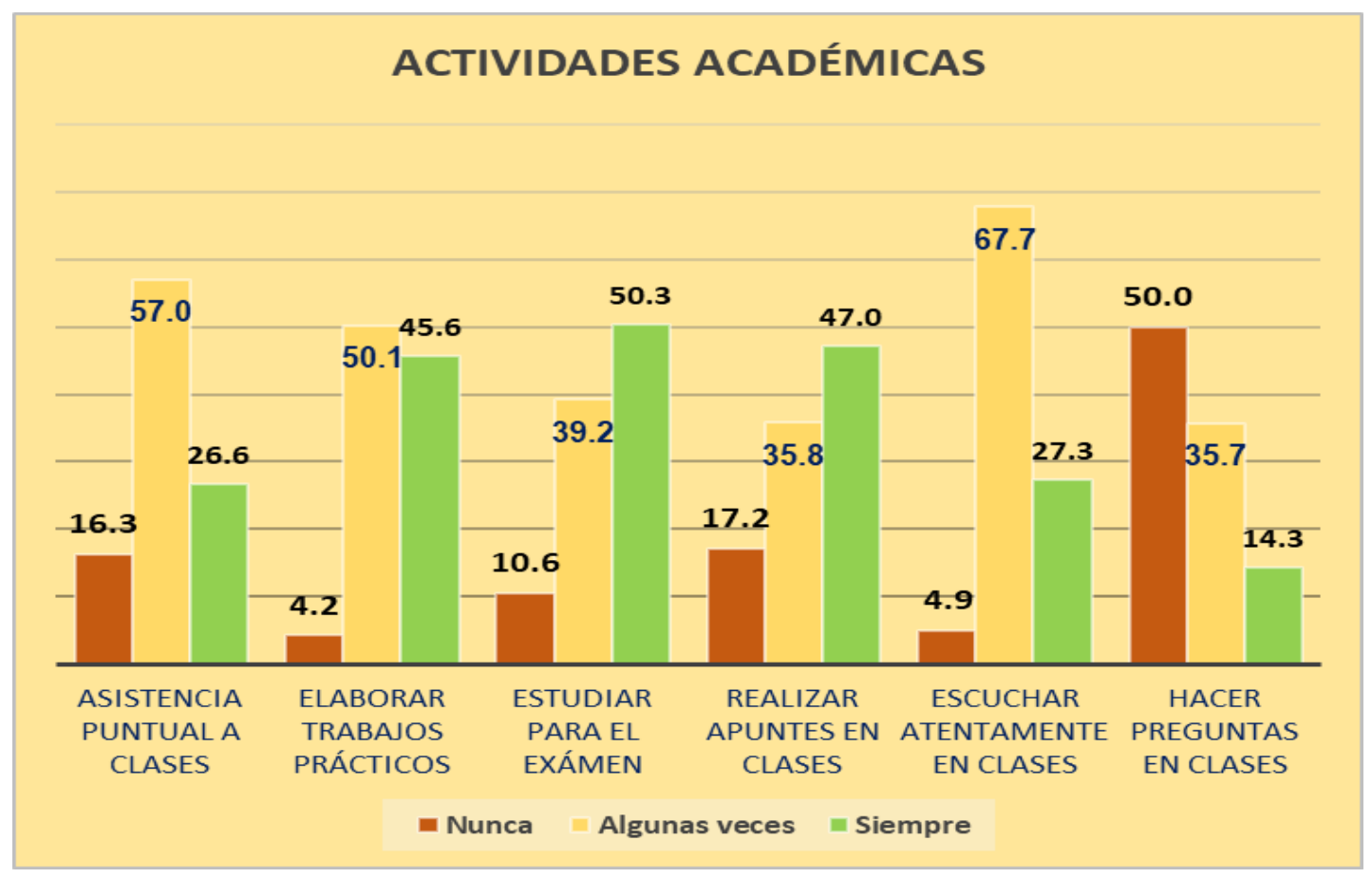

Figura 1. Realización de actividades de desempeño académico 
La mayoría (67.7\%) señala que solo algunas veces escucha atentamente en clases; en segundo lugar, señalan que siempre estudian para el examen (50.3\%), realizan apuntes en clase $(47 \%)$ y la mayoría señala que nunca hace preguntas en clases (50\%).

Seguidamente la figura 2 refleja datos sobre las asignaturas consideradas como difíciles de aprender para los estudiantes. En donde se destacaron las siguientes asignaturas: Estadística, Investigación Educativa, Educación Virtual, Creática y Pedagogía General.

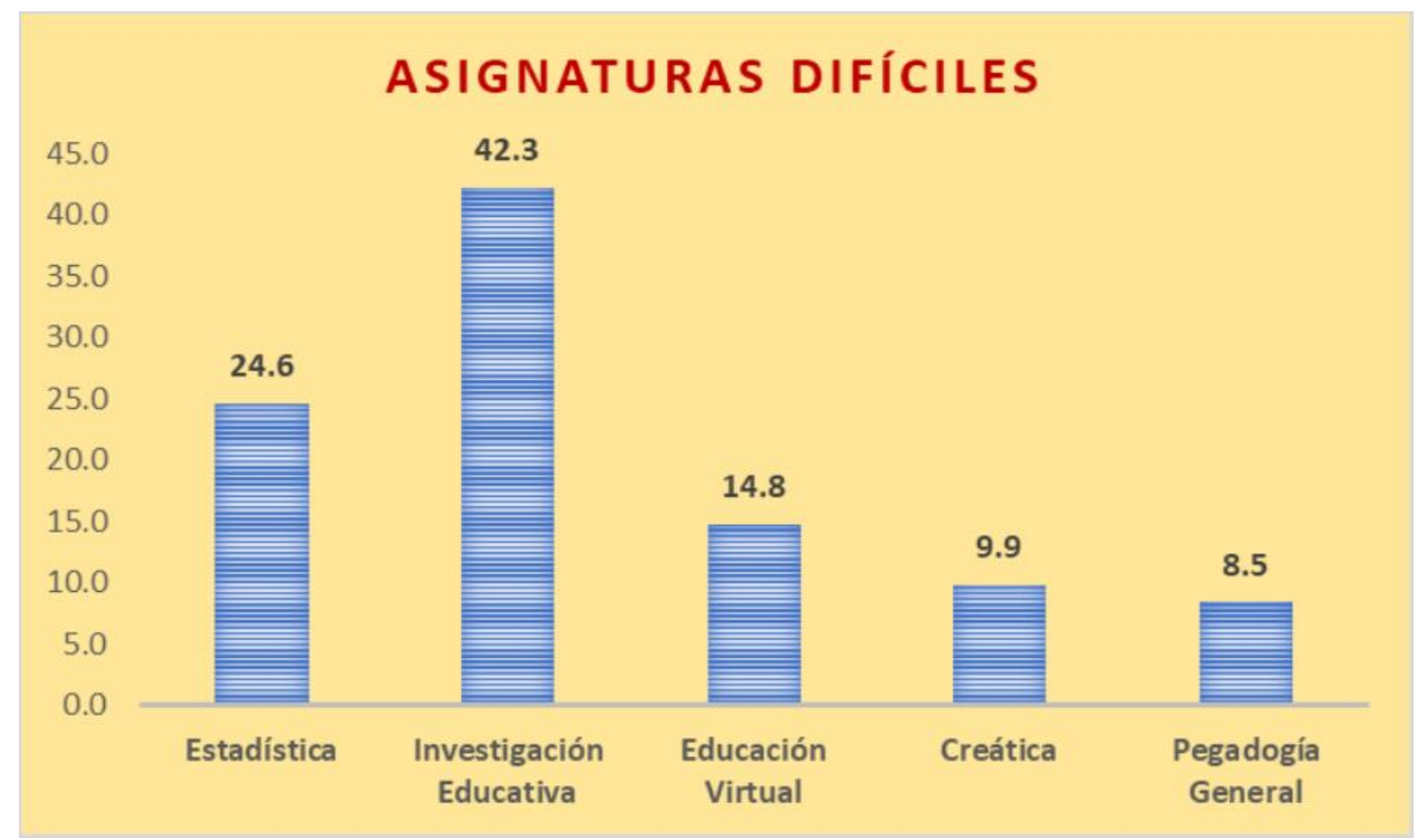

Figura 2. Asignaturas consideradas como difíciles de aprender

En relación con las cinco asignaturas, la Investigación Educativa con el $42.3 \%$ es considerada como la más difícil, en segundo lugar está la asignatura de Estadística con un $24.6 \%$, y por último la asignatura denominada Educación virtual con un $14.8 \%$, siendo estas tres las más difíciles.
En la figura que sigue se muestran los datos relacionados a los factores que influyen en el aprendizaje de la asignatura Investigación Educativa: sociocultural, económico, automotivación, motivación docente, técnicas de estudio y estrategias de enseñanza. 


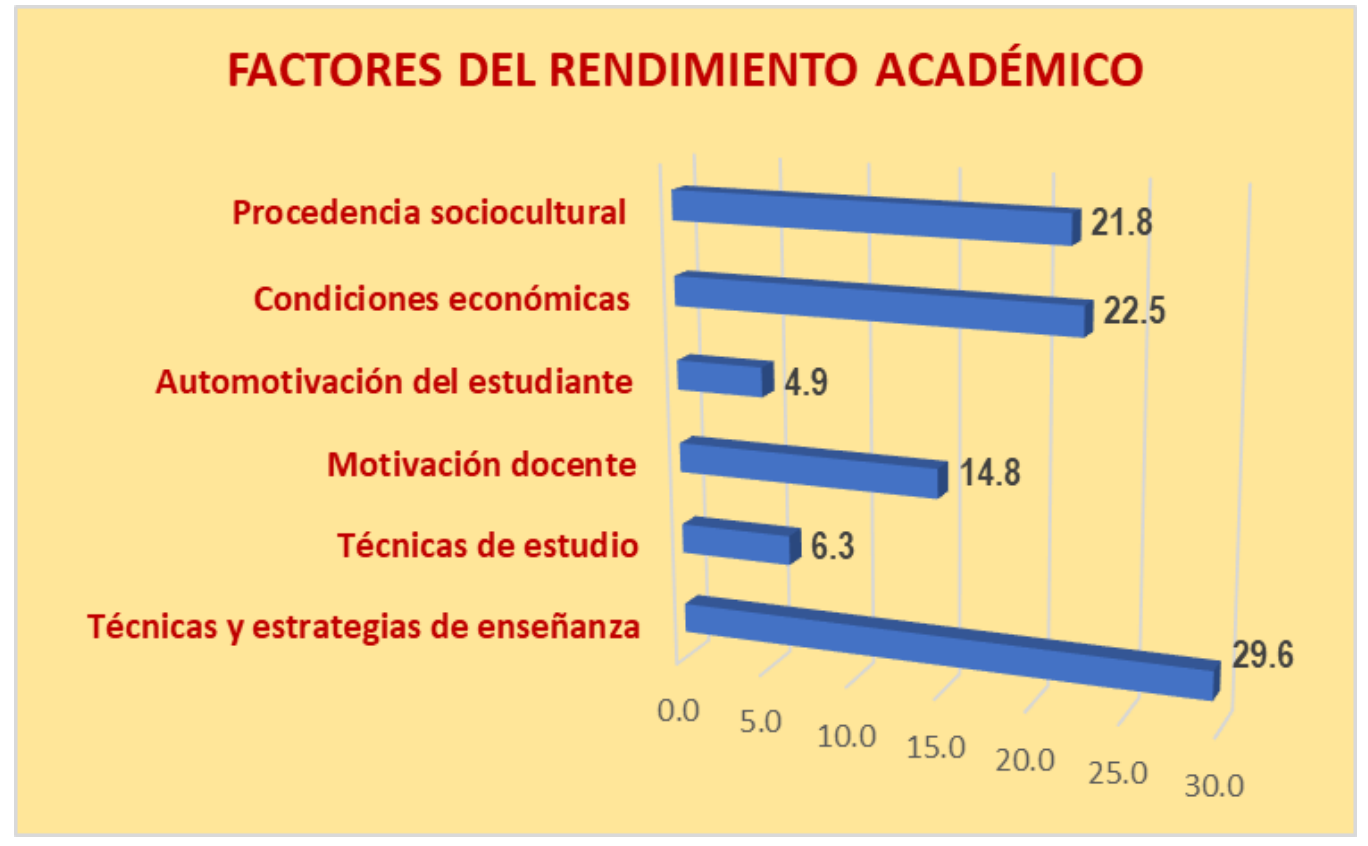

Figura 3. Factores que influyen en el aprendizaje de la asignatura Inv. Educativa

Los factores más relevantes del rendimiento académico constituyen las técnicas y estrategias de enseñanza (29.6\%), en segundo lugar, las condiciones económicas (22.5\%) y en tercer lugar la procedencia sociocultural $(21.8 \%)$.

\section{Resultados cuantitativos según revisión documental}

Con relación a la frecuencia de datos según rangos de calificaciones se consiguió la siguiente información que destaca que entre 51-66 es bajo, 67-83 es medio y 84-100 es alto. (Figura 4)

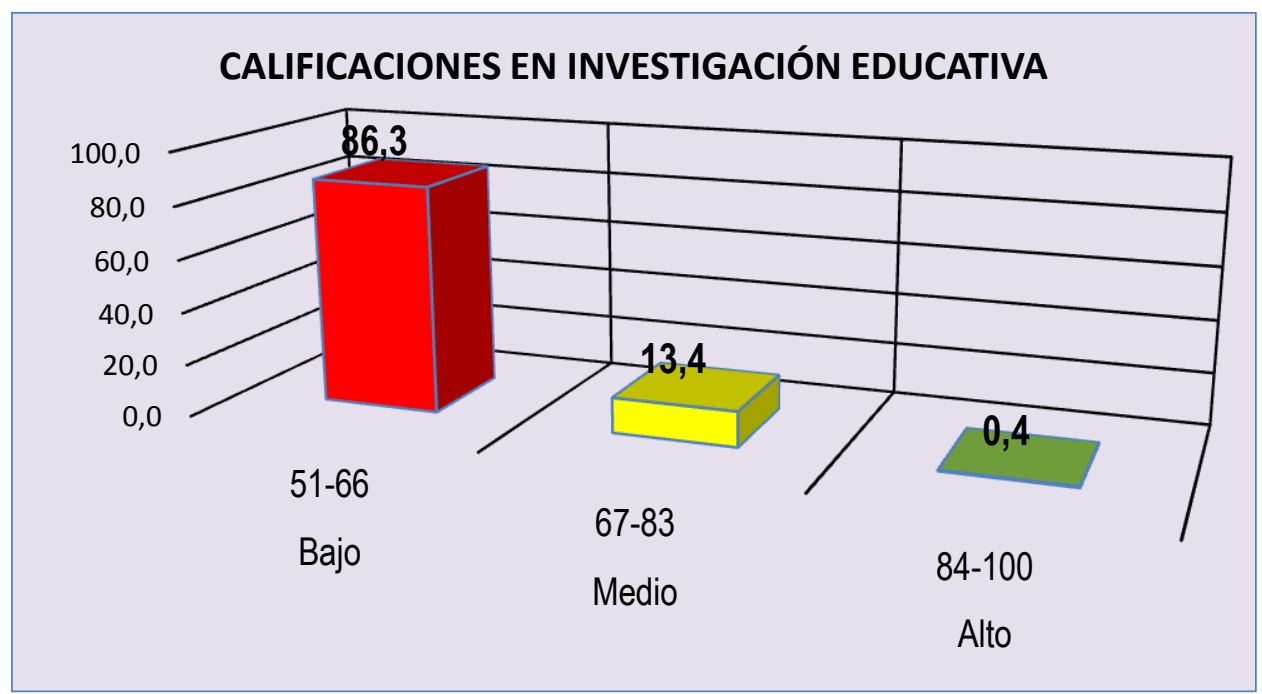

Figura 4. Nivel de rendimiento en la asignatura de Investigación Educativa 
En esta Figura 4 se observa que el $86.3 \%$ tiene un rendimiento en el rango de 51-66, un $13.4 \%$ en el rango de $67-83$ y solo el $0,4 \%$ llega a un rango de $84-100$, correspondiente a las calificaciones de la asignatura de Investigación Educativa.

\section{Resultados cualitativos según observación en aula}

Las observaciones en aula se realizaron clasificados en tres etapas: al inicio, en el desarrollo y al finalizar las clases académicas, cuyos registros importantes se describen en la siguiente Tabla 2.

Tabla 2. Observación del proceso de enseñanza aprendizaje en el aula

\begin{tabular}{|c|c|c|}
\hline ETAPAS & INDICADORES & REGISTRO DATOS (ITEMS) \\
\hline Inicio & $\begin{array}{l}\text { 1. Ambiente físico del } \\
\text { aula } \\
\text { 2. Interacción docente } \\
\text { con los estudiantes } \\
\text { 3. Presentación de la } \\
\text { clase }\end{array}$ & $\begin{array}{l}\text { 1. Las aulas tienen pizarra acrílica, dispositivo para data } \\
\text { y la laptop que los docentes poseen. } \\
\text { 2. Los docentes llegan con un leve retraso, se saludan, } \\
\text { difícilmente demuestra entusiasmo, amabilidad y } \\
\text { empatía. } \\
\text { 3. Presentan la temática y no así los objetivos de la } \\
\text { clase. }\end{array}$ \\
\hline Desarrollo & $\begin{array}{ll}\text { 4. } & \text { Postura docente } \\
\text { 5. Expresión oral } \\
\text { 6. Uso de recursos } \\
\text { audiovisuales } \\
\text { 7. Uso de habilidades } \\
\text { persuasivas } \\
\text { 8. Uso de técnicas } \\
\text { interactivas } \\
\text { 9. Tipo o estilo docente } \\
\text { 10. Tipo de estudiante }\end{array}$ & $\begin{array}{l}\text { 4. El docente se mueve, utiliza lenguaje corporal, gestos } \\
\text { y contacto visual, pero de manera muy escasa. } \\
\text { 5. El docente se expresa con claridad y no pierde el hilo, } \\
\text { pero muy pocas veces usa palabras sencillas } \\
\text { 6. Usan data display, pero no usa los videos } \\
\text { 7. Para persuadir a los estudiantes, el docente no utiliza } \\
\text { anécdotas, consejos, frases, chistes, ilustraciones, etc. } \\
\text { 8. También hace poco uso de técnicas grupales, como } \\
\text { ser: dinámicas, juegos, recursos prácticos, etc. } \\
\text { 9. Los docentes observados reflejan un estilo exigente, } \\
\text { tradicional y nada participativo ni práctico. } \\
\text { 10. Los estudiantes reflejan pasividad porque no } \\
\text { preguntan, solo escuchan y se limitan a realizan } \\
\text { apuntes, etc. }\end{array}$ \\
\hline Finalización & $\begin{array}{l}\text { 11. Síntesis } \\
\text { 12. Retroalimentación } \\
\text { 13. Tareas prácticas }\end{array}$ & $\begin{array}{l}\text { 11. No expresa conclusiones sobre los puntos } \\
\text { desarrollados } \\
\text { 12. No manifiesta retroalimentación ni criterios de } \\
\text { mejoramiento } \\
\text { 13. Instruye tareas individuales para la próxima clase. }\end{array}$ \\
\hline
\end{tabular}


En la parte inicial, las aulas cuentan con mínimos dispositivos tecnológicos, los docentes llegan con cierto retraso, interactúan muy brevemente y presentan el tema, pero no explican el objetivo de aprendizaje. En el desarrollo de las clases, la postura docente es más hablado que expresión corporal y gestual; hacen el uso de data display, pero no usan las dinámicas interactivas, el docente hace una exposición con estilo tradicional. En los estudiantes se percibe la falta de preguntas y opiniones por cuenta propia, que reflejan un estilo de enseñanza y aprendizaje tradicionales.

\section{Resultados cualitativos según análisis de contenido}

En el análisis de contenido de las redacciones elaboradas por los estudiantes sobre el tema "Mi rendimiento académico", los resultados se han categorizado según los niveles y sus indicadores descritos anteriormente en la parte metodológica.

Las siguientes redacciones contienen datos en su nivel inferior:

1. "Mi rendimiento académico en la asignatura investigación no es el mejor porque no hay buenos docentes, utilizan palabras desconocidas, no motivan a los estudiantes ni utilizan técnicas adecuadas. desanimado".

2. "Prefiero no decir nada sobre mi rendimiento en investigación, creo que la carrera no es para mi, solo estoy asistiendo porque mi papá quiere que estudie una profesión."

3. "El rendimiento académico en la asignatura investigación, es parte de la educación y las personas sin educación están destinados al fracaso, pero como Bolivia no tiene una buena política educativa, está dificil."

4. "No pensaba estudiar esta carrera y me esta yendo mal, no se si es mis capacidades o la forma de enseñar que tiene el docente.

Figura 5. Contenidos de redacción en su nivel inferior

Las redacciones reflejan pérdida de interés por el desempeño académico, no hacen casi ningún esfuerzo para mejorar su rendimiento académico e inclusive han perdido el interés por la formación profesional y desanimados buscan cambiar de carrera o simplemente piensan dejar de estudiar.

En las siguientes redacciones se observan contenidos en su nivel medio: 
1. "Una de mis metas es salir profesional. Doy todo de mi para tener buenas notas notas en la materia investigación, pero no siempre salen bien las cosas, hay dificultades y tropiezos:.

2. "Muchas veces influye el docente, cuán amena sean sus clases, la tonalidad de su voz, oratoria, dominio de temas; en si no se ve en ningún docente estas caracteristicas...

3. "Lo importante es que cuando tengo buenas notas me anima a estudiar más, pero cuando mis notas son bajas me siento muy triste y a veces ya no me dan las ganas de ir a clases; pero lo importante es que llegue a ser un profesional para trabaiar v tener el sustento económico anhelado"

Figura 6. Contenidos de redacción en su nivel medio

Las expresiones se caracterizan por ser ambivalentes, dependientes y propósitos limitados a culminar los estudios profesionales, porque algunas veces se sienten bien y otras veces triste, tienen interés por las actividades prácticas, pero no las demuestran, hacen esfuerzos, pero no lo hacen por cuenta propia y perciben la profesión como un medio de sustento económico, deseo de logro y prestigio.

En las redacciones se pueden apreciar contenidos en su nivel superior:

1. "Mi rendimiento académico en investigación es muy satisfactorio porque una de las materias claves es salir de las dudas preguntando. Mi sueño es lograr por lo menos 2 ó 3 carreras, no solo para la satisfacción personal ni para que mis papás se sientan bien, sino que con mi profesión pueda ser útil a la sociedad".

2. "Bueno, primeramente, doy todo de mi en el estudio, porque estoy segura de que seré una buena profesional porque aprendo muchas cosas aquí. Lo único que me disgusta son las marchas porque son perjudiciales; sin embargo, en los momentos de paros y marchas, conscientemente estudio estudio y leo libros que me ayudarán..."

3. "Soy madre soltera, tengo un hijo de 3 años y me gusta la carrera. Ante cualquier circunstancia, considero que es importante esforzarse para salir adelante y estudiar con optimismo. Quiciera ser una cientista en educación, no solo para decir aue sov profesional sino para avudar a la aente necesitada..."

Figura 7. Contenidos de redacción en su nivel superior 
Según la figura 7, el contenido de las expresiones en su nivel superior manifiestan satisfacción, optimismo y proactividad, más que limitarse a la culminación profesional se proyectan al futuro ejercicio profesional $y$, principalmente, perciben su rendimiento académico como un medio para su adecuada formación profesional y consideran la profesión como instrumento solidario con las necesidades sociales, lo que va más allá de utilizar la profesión como medio de sustento económico y prestigio personal.

\section{Discusión}

A partir de los datos de la encuesta, se percibe que la capacidad de atención del estudiante es importante durante el desarrollo de las clases académicas, así mismo muchos otros factores como motivación, interés, condiciones económicas, situación laboral del estudiante, los problemas personales, etc. Pero, por otro lado, está relacionado con la falta de un ambiente académico ameno, interactivo y vivencial. En los ambientes de clases tradicionales, generalmente los estudiantes hacen las cosas por exigencia y se limitan a realizar apuntes, no preguntan ni aportan ideas porque no tienden a ser proactivos. Este fenómeno puede estar relacionado con la trascendencia histórica, sociocultural e identidad aimara cuyos rasgos de carácter tienden a ser poco proactivos y más dependientes debido a la herencia cultural de las generaciones antepasadas.

Con respecto al factor sociocultural, es como si el estudiante heredara el carácter dependiente y las consideraciones de baja autoestima de acuerdo con el mecanismo de identidad cultural, que también constituyen condiciones psicológicas que influyen automáticamente en el rendimiento académico, porque las bajas valoraciones hacia sí mismo y el carácter de dependencia hacia los demás, restringen las posibilidades de autodeterminación.

Las condiciones económicas, si bien no repercuten directamente, influyen en la condición de eficaz desempeño del estudiante, como ser: carencia de alimentación, la necesidad de trabajar, las facilidades de compra de materiales o libros y el acceso al uso de recursos tecnológicos.

Además, las estrategias de enseñanza tradicionales tienen que ver con la forma de organización de clases de índole memorística, individualista, teoricista y la falta de significación sistémica en el proceso de enseñanza aprendizaje de la asignatura Investigación Educativa.

De acuerdo con el análisis de contenido de las calificaciones, más que los bajos resultados, es más preocupante que casi ninguno alcanza los puntajes altos. Esto refleja que no hay mejores estudiantes en la asignatura de Investigación Educativa, siendo la construcción del conocimiento una de las funciones importantes del profesional en Ciencias de la Educación. Por lo tanto, estos resultados no solo hacen que percibamos el tipo de profesional que el estudiante universitario quiere ser, sino la calidad de formación profesional que la Universidad está entregando a la sociedad boliviana. Este resultado contrasta con la visión de la carrera Ciencias de la Educación, porque se considera un "...Referente de formación profesional en el área de la educación, priorizando la investigación científica en todos los campos del conocimiento, aplicando a la práctica para la transformación económica, social, cultural y política..." (UPEA, 2015, p. 75)

Según resultados de la observación en las aulas, el proceso de enseñanza aprendizaje de la asignatura Investigación Educativa no se percibe como una experiencia concerniente a 
procesos de aprendizaje significativo, ni colaborativo ni vivencial. Esta situación podría estar relacionado con la falta de ciertas estrategias como ser: la aplicación de dinámicas interactivas, trabajo en equipo, aprender haciendo y otros; sin embargo, debido a que tales estrategias implican mayor uso de tiempo para desarrollar las clases, los docentes prefieren abarcar más contenidos en menor tiempo y, por ello, se limitan a realizar clases propios del estilo tradicional. Además, la posibilidad de transitar de un paradigma teoricista hacia un enfoque práctico y vivencial no es cuestión de querer transformar de noche a la mañana, sino que implica identificar contenidos inadecuados del proceso enseñanza aprendizaje tradicional para superarlos $\mathrm{y}$, a su vez, identificar o innovar estrategias alternativas más eficientes que constituyen un enfoque de enseñanza aprendizaje diferente, consistente y transformador

De acuerdo con los criterios de análisis de contenido de las redacciones que elaboraron los propios estudiantes, se han categorizado tres niveles de percepción del rendimiento académico en la asignatura de Investigación Educativa: nivel inferior, medio y alto.

- Nivel de rendimiento inferior. Es producto de las condiciones socioculturales, históricas, económicas y el enfoque de educación tradicional; por otra parte, estas experiencias académicas desfavorables influyen en el bajo desempeño cognoscitivo, en la insatisfacción con respecto a la asignatura e inclusive la carrera profesional, en la pasividad de las acciones, desinterés o desmotivación, perdida de la voluntad y carencia en la convicción profesional emprendida.
- Nivel de rendimiento medio. Denota el aparente esfuerzo y la motivación para lograr mejores resultados cognoscitivos y los hábitos prácticos que ayuden a estudiar por cuenta propia, pero de manera dependiente y exigencia docente. Por consiguiente, los estudiantes que se encuentran en este nivel tienen amplias oportunidades de mejorar su nivel de rendimiento académico a partir de estrategias necesarias como ser: el aprendizaje más significativo, más colaborativo que el logro egoísta y aprendizajes más prácticos que teóricos.

- Nivel de rendimiento alto. Son muy pocos los estudiantes que reflejan este nivel, por su dificultad y complejidad, porque lograr estos niveles ideales implican realmente mayores esfuerzos como para superar factores adversos; además los esfuerzos no son suficientes, son necesarias alcanzar un nivel de reflexión consciente y la convicción en la formación profesional que contemple una plena satisfacción y persistencia, de modo que los obstáculos no sean factores que desestabilicen la personalidad del estudiante, sea capaz de estudiar por cuenta propia $\mathrm{y}$, más que todo, comprenda la necesidad de hacer intenciones colaborativas en vez de procurar acciones egoístas de aprendizaje.

\section{Propuesta: estrategia metodológica}

La estrategia metodológica es un sistema de acciones a corto, mediano y largo plazo que permite la "transformación del proceso de enseñanza aprendizaje tomando como base los métodos y procedimientos para el logro de los objetivos determinados en un tiempo concreto". (Rodríguez del Castillo, 2014, p. 25) 
En este sentido, la estrategia metodológica que se propone para mejorar las competencias investigativas está organizada en base a la siguiente estructura: Fundamentación teórica, diagnóstico del objeto, objetivo de la estrategia, planificación de la estrategia, instrumentación y evaluación.

\section{Fundamentación teórica}

La estrategia metodológica aquí propuesto se fundamenta en tres enfoques teóricos del proceso enseñanza aprendizaje: enfoque significativo, colaborativo y vivencial.

- El aprendizaje significativo. "Es el proceso a través del cual una nueva información (un nuevo conocimiento) se relaciona de manera no arbitraria y sustantiva (no-literal) con la estructura cognitiva de la persona que aprende". Cuando un nuevo conocimiento se vincula con otra anterior cobra mayor consistencia porque se vincula y se sustenta con la información ya existente. (Salazar, 2017, p. 43)

- El aprendizaje cooperativo. "Permite al estudiante participar activamente de su propio aprendizaje y el de sus compañeros." (Linares, 2017, p. 19). Por lo tanto, cuando un estudiante tiene la oportunidad de ser tomado en cuenta y el tomar el compromiso de aportar conocimientos en favor de los demás, le permite mayores condiciones motivacionales para mejorar el propio aprendizaje, según manifiesta.

- Aprendizaje vivencial. Según Marcillo, y otros. (2019), El "aprendizaje experiencial consiste en generar espacios que posibiliten la vivencia, que puedan ser sucedidos por momentos de reflexión para que dicha vivencia se convierta en experiencia"; quiere decir que cuando se aprende en la experiencia vivida es más consistente y significativo el proceso de apropiación de los conocimientos en los estudiantes; además no se trata simplemente de aplicar la teoría sino el generar la reflexión teórica a partir de la experiencia vivida. (p. 50).

\section{Diagnóstico del objeto}

El diagnóstico consiste en la descripción de la situación actual del objeto problema como vía para transformar en una situación futura de problema resulto. Tal cual señala Rodríguez del Castillo (2014) cuando menciona que el diagnóstico: "Indica el estado real del objeto y evidencia el problema en torno al cual gira y se desarrolla la estrategia." (p. 22).

En este caso, el problema descrito anteriormente en esta investigación es el bajo rendimiento académico en la asignatura Investigación Educativa, vinculado a los factores internos y externos de la personalidad del estudiante.

Los factores internos de la personalidad tienen que ver con las propias manifestaciones de: la insatisfacción, pasividad y pérdida de interés por proseguir la asignatura y la carrera misma en proceso de formación; aunque quienes manifiestan su interés y esfuerzo está limitado a los objetivos inmediatos de lograr conseguir un título para luego dedicarse a ganar dinero y ostentar el prestigio anhelado, lejos de considerar el encargo social de contribuir estrategias de solución a los problemas sociales.

\section{Objetivo de la estrategia}

Considerando que el objetivo de la estrategia es el polo opuesto de la situación del problema, o sea es la solución al problema que se espera después de la aplicación de la estrategia metodológica a través del proceso de 
enseñanza aprendizaje. En esta comprensión, el objetivo principal describe la siguiente alternativa:

Contribuir al desarrollo de las competencias en el campo de la Investigación Educativa a nivel de conocimientos, actitudes y habilidades, a través del uso de las dinámicas interactivas y vivenciales en grupo, en favor de los estudiantes de la carrera Ciencias de la Educación de la Universidad Pública de El Alto (UPEA).

\section{Planificación estratégica}

Para la planificación estratégica es necesario que el objeto de estudio este descrito con claridad, puesto que es fundamental responder a la pregunta ¿qué se enseña? y ¿qué se aprende? en este proceso de enseñanza aprendizaje.

En esta comprensión, el objeto estratégico consiste en la enseñanza y aprendizaje de la asignatura Investigación Educativa; lo que en esta propuesta se delimita al aprendizaje del diseño del perfil de investigación, es decir orientado a cómo desarrollar las competencias investigativas a nivel de perfil, que comprende siete etapas, sus contenidos y sus dinámicas como se describen en la siguiente tabla:

Tabla 3. Etapas, contenidos didácticos y dinámicas.

\begin{tabular}{|c|c|c|}
\hline ETAPAS & CONTENIDOS & DINÁMICAS \\
\hline $\begin{array}{l}1 \\
\text { Elección del tema de } \\
\text { investigación }\end{array}$ & $\begin{array}{l}\text { - ¿Qué aspecto educativo investigar? } \\
\text { - ¿En qué grupos de personas? } \\
\text { - ¿En qué lugar? }\end{array}$ & La caja de ideas \\
\hline $\begin{array}{c}2 \\
\text { Delimitación del objeto }\end{array}$ & $\begin{array}{l}\text { - } \text { Carrera } \\
\text { - Área } \\
\text { - } \quad \text { Componentes de área }\end{array}$ & La botella preguntona \\
\hline $\begin{array}{l}3 \\
\text { Planteamiento del } \\
\text { problema }\end{array}$ & $\begin{array}{l}\text { - Causas negativas } \\
\text { - Consecuencias negativas }\end{array}$ & $\begin{array}{l}\text { Desarraigando el árbol } \\
\text { seco }\end{array}$ \\
\hline $\begin{array}{l}4 \\
\begin{array}{l}\text { Formulación de } \\
\text { objetivos }\end{array}\end{array}$ & $\begin{array}{l}\text { - Factores positivos } \\
\text { - Consecuencias positivas }\end{array}$ & $\begin{array}{l}\text { Fortaleciendo el árbol } \\
\text { verdoso }\end{array}$ \\
\hline $\begin{array}{c}5 \\
\text { Índice del marco teórico }\end{array}$ & $\begin{array}{l}\text { - } \quad \text { Concepto y significado } \\
\text { - } \text { Componentes y relaciones } \\
\text { - Niveles o grados } \\
\text { - } \quad \text { Estructura y función }\end{array}$ & Mis objetos favoritos \\
\hline
\end{tabular}


ETAPAS

DINÁMICAS

\begin{tabular}{|c|c|c|}
\hline $\begin{array}{l}6 \\
\text { Formulación de la } \\
\text { hipótesis }\end{array}$ & $\begin{array}{ll}\text { - } & \text { Componentes y relaciones del objeto } \\
\text { - } & \text { Afirmación anticipada } \\
\text { - } & \text { Idea de solución al problema }\end{array}$ & $\begin{array}{l}\text { Razonando con los } \\
\text { globos }\end{array}$ \\
\hline $\begin{array}{l}7 \\
\text { Determinación del } \\
\text { método }\end{array}$ & $\begin{array}{ll}\text { - } & \text { Enfoque metodológico } \\
\text { - } & \text { Métodos teóricos y empíricos } \\
\text { - } & \text { Técnicas e instrumentos } \\
\text { - } & \text { Población y muestra }\end{array}$ & $\begin{array}{l}\text { Feria de alimentos, } \\
\text { animales y objetos }\end{array}$ \\
\hline
\end{tabular}

\section{Instrumentación}

La instrumentación consiste en la organización de espacios, tiempo, responsables y participantes.

- Espacio. Considerando que es posible "Una didáctica externa a la escuela, pero comprometida con la formación de nuevos ciudadanos", se plantea trabajar en espacios extra-aula porque las interacciones $y$ vivencias son más apropiadas en tales espacios, además el enfoque estratégico plantea trabajar desde las vivencias que ocurren más en espacios externos a las aulas universitarias; aunque no se excluye trabajar al interior de las aulas. (Bolaños, Castellanos y Rojas, 2019, p.15).

- Tiempo. También es necesario plantear se trabaje en tiempos extracurriculares y curriculares, porque los componentes del objeto estratégico anteriormente planteados no deben interferir a la estructura curricular planificada previamente; más bien es necesario complementar e integrar aquellos aspectos necesarios con los componentes curriculares.

- Responsabilidades. Las responsabilidades para la aplicación de esta estrategia se organizarán a través de una comisión, ya que el enfoque mismo plantea la necesidad de trabajo en equipo más que individual.

- Participantes. Entre los participantes se organizarán comisiones conformadas por las siguientes personas: Investigador, docentes de la asignatura de Investigación Educativa y estudiantes de los dos últimos semestres de la gestión académica que tengan interés en elaborar sus investigaciones.

\section{Evaluación}

Según Fuentes, (2009) el sistema de evaluación debe contribuir a fomentar la capacidad crítica, autocrítica, independencia y creatividad en los estudiantes, por lo que éste debe caracterizarse por:

- Un elevado carácter participativo y constructivo...

- Potenciar los niveles de desarrollo de la formación del profesional, a través de cambios cualitativos...

- Fomentar el desarrollo de un pensamiento investigativo, crítico, reflexivo, transformador e independiente...

- Promover la consolidación del proceso de enseñanza-aprendizaje como un espacio 
de construcción de significados y sentidos...

- Contribuir al desarrollo de un ambiente donde la evaluación no sea vista como un proceso que inspire temor a los estudiantes. (pp. 253-254)

La evaluación es realizable en tres fases importantes: al inicio, en el proceso y al final.

- Evaluación inicial. Implica una evaluación diagnóstica, en términos de pre-test, antes de iniciar los módulos del desarrollo del programa.

- Evaluación en proceso. Consiste en las valoraciones y reflexiones críticas al final de cada sesión temática y al terminar cada módulo desarrollado.

- Evaluación final. En esta fase de evaluación se realizará una verificación de resultados al finalizar la aplicación del programa, en términos de pos-test, con el propósito de validar el programa ejecutado y posterior enriquecimiento.

\section{CONCLUSIÓN}

De acuerdo con la investigación realizada, el bajo rendimiento académico de los estudiantes en la asignatura Investigación Educativa tiene relación con dos aspectos esenciales: la dificultad que experimentan los estudiantes al aprender y la coherencia práctica de los contenidos teóricos asimilados. Y, como factores principales, resaltan las técnicas o dinámicas interactivas de enseñanza aprendizaje, condiciones económicas y la procedencia sociocultural aimara.

El bajo rendimiento académico involucra estudiantes, docentes y el contexto en el que se encuentran. El interés, la autovaloración y la motivación con que proyectan su formación profesional reflejan los problemas históricoculturales que han vivido sus antepasados. Estos factores repercuten principalmente en la visión limitada de la profesión como simple instrumento para el ejercicio laboral con fines económicos y las aspiraciones egoístas, sin considerar el encargo social de la profesión como servicio.

Por otra parte, el bajo rendimiento tiene relación con el desempeño docente: uso de técnicas y estrategias interactivas de enseñanza, la vigencia del enfoque tradicional y la complejidad de una enseñanza desde la práctica.

La fundamentación teórica del proceso de enseñanza aprendizaje refiere tres teorías importantes: aprendizaje significativo, colaborativo y vivencial. El aprendizaje significativo, en su connotación actual holística y holográfica, los saberes actuales integran y representan a su vez saberes previos. El aprendizaje colaborativo implica trabajo en equipo, el esfuerzo de dejar el aprendizaje egoísta y el compromiso de aprender unos por otros o en términos dialécticos el aprender enseñado y enseñar para aprender. Y, el aprendizaje vivencial, que aporta tres importantes elementos: lo dinámico, activo y creativo, lo que implica que el aprendizaje vivencial concierne un proceso de enseñanza-aprendizaje a partir de la experiencia vivida, la interacción colaborativa y fundamentalmente motiva el desarrollo de las capacidades creativas.

La propuesta construida en esta investigación constituye una estructura que articula seis componentes: El bajo rendimiento académico como problema, la mejora de rendimiento académico como objetivo, el proceso de enseñanza aprendizaje de la asignatura de Investigación Educativa como objeto, la estrategia metodológica como 
contenido; aprendizajes significativo, colaborativo y vivencial como método, incluyendo las dinámicas interactivas como forma y medio; y finalmente, el componente resultado como competencia investigativa en su dimensión cognitiva, afectiva y práctica; y su respectiva evaluación.

\section{REFERENCIAS}

Álvarez de Zayas, C. M. (2016). Epistemología del caos. Tercera edición, Cochabamba, Bolivia: Grupo Editorial Kipus

Álvarez de Zayas, Carlos M. y Sierra Lombardía, V. Virginia, (2019). Metodología de la investigación científica. Novena edición, Cochabamba, Bolivia: Grupo Editorial Kipus

Bolaños Motta, J. I., Castellanos Sánchez, M. T., y Rojas Gómez, J. O. (2019). Didáctica de la ética. El aporte de Mockus a la gobernabilidad en Colombia. Hallazgos, 16(32),

151-167. https://dx.doi.org/10.15332/2422409x.4 817

Carbonell, E., (2009). Relación entre la motivación profesional del docente y la satisfacción de los estudiantes en la formación pacicultora. Recuperado de http://www.monografias.com/trabajos69 /relacion-motivacion-docentessatisfaccion-estudiantes/relacionmotivacion-docentes-satisfaccionestudiantes $2 . \mathrm{shtml}$

Fuentes, H. (2009). Pedagogía y didáctica de la educación superior. Santiago de Cuba, Universidad de Oriente, Centro de Estudio de Educación Superior "Manuel F. Gran”
Linares, A. E. (2017). El aprendizaje cooperativo y su influencia en el rendimiento académico en el área de matemática de los alumnos de educación secundaria. Recuperado de http://www.imes.edu.uy/new/wpcontent/uploads/2017/05/PUBLICAS/M ONOGRAFIA\%204246\%2002.pdf

Marcillo, M.F., Veloz, E.J., Solís, M., Haro, A.E (2019). Aprendizaje experiencial y su aplicación en el proceso de enseñanza y aprendizaje. Recuperado de: http://142.93.18.15:8080/jspui/bitstrea $\mathrm{m} / 123456789 / 312 / 1 /$ Aprendizaje $\% 20 \mathrm{E}$ xperiencial\%20\%20y\%20su\%20Aplicaci \%C3\%B2n\%20en\%20el\%20Proceso $\% 20$ de\%20Ensenanza\%20y\%20Aprendizaje.p df

Rodríguez del Castillo, M. A. (2014). La estrategia como resultado científico de la investigación educativa. Santa Clara, Villa Clara, Cuba

Salazar, J. A. (2017). El aprendizaje significativo y su relación con el uso de las TIC en la enseñanza de la informática de los estudiantes del grado noveno de la institución educativa sagrado corazón de paz de Ariporo Casanare. Recuperado de: http://repositorio.uwiener.edu.pe/bitstre am/handle/123456789/1788/MAESTRO $\% 20$ -

$\% 20$ Salazar $\% 20$ Fuentes $\% 2$ C $\% 20 \% 20$ Jai me\%20Augusto.pdf?sequence=1\&isAllow ed $=y$

Universidad Pública de El Alto (UPEA), (2015). Rediseño curricular. Jornadas académicas. Ciudad de El Alto, Bolivia, 14982 